\title{
Turbulence Modelling for RANS CFD analyses of Multi-Nozzle Annular Jet Pump Swirling Flows
}

\author{
A. Morrall ${ }^{\mathrm{a}, *}$, S. Quayle ${ }^{\mathrm{a}}$, M.S. Campobasso ${ }^{\mathrm{a}}$ \\ ${ }^{a}$ University of Lancaster, \\ Department of Engineering. Engineering Building, Gillow Avenue, \\ Lancaster LA1 $4 Y W$, United Kingdom.
}

\begin{abstract}
This study focusses on the fluid mechanic analysis and performance assessment of a one-phase swirling flow multi-nozzle annular jet pump using Reynoldsaveraged Navier-Stokes simulations and experimental measurements carried out with a bespoke test rig. The numerical investigation of the flow physics of the device, key to understanding its fluid dynamics and optimising its performance, is made particularly challenging by the existence of flow swirl. Thus, the predictive capabilities of two alternative approaches for the turbulence closure of the Reynolds-averaged Navier-Stokes equations, namely the $k-\omega$ shear stress transport and the Reynolds stress models, are assessed against measured static pressure fields for three regimes characterised by different swirl strength, and a thorough cross-comparative analysis of the flow physics using the two closures is performed to complement the information provided by the experimental measurements. At the lowest swirl level, the two simulation types are in very good agreement, and they both agree very well with the measured static pressure fields. As the flow swirl increases, the two numerical results differ more and the Reynolds stress model is in better agreement with the measured static pressure. At the highest swirl level the shear stress transport analysis predicts weaker dis-
\end{abstract}

${ }^{\star}$ Fully documented templates are available in the elsarticle package on CTAN

* Corresponding author

Email addresses: a.morrall1@lancaster.ac.uk (A. Morrall), s.quayle@lancaster.ac.uk (S. Quayle), m.s.campobasso@lancaster.ac.uk (M.S. Campobasso)

Preprint submitted to Journal of ${ }^{A} T_{E} X$ Templates

July 23, 2020 
sipation of the jet energy and stronger mixing of injected and pumped streams, resulting in higher performance predictions than obtained with the Reynolds stress model. A CFD-based sensitivity analysis also highlights the impact of nozzle diameter and flow swirl on the pump performance, providing new guidelines for the design optimisation of this pump.

Keywords: Jet pumps, Reynolds-Averaged Navier-Stokes CFD, Eddy viscosity turbulence models, Reynolds Stress Models, Experimental validation.

\section{Introduction}

A jet pump is a fluidic device that has no internal moving parts, and instead drives media by means of turbulent mixing and energy exchange between two fluid streams, the motive or primary stream and the pumped or secondary stream. The phase of either stream can be gaseous or liquid, and the two fluids may also be different. In some cases, the secondary stream can also be a mix of fluids or contain solid particles. The motive fluid is pressurised and discharged from one or more nozzles into the bore of the pump as a high velocity jet. The velocity gradient of the jet draws the secondary fluid through viscous forces, with the secondary fluid entrained through turbulent mixing and energy exchange. This process results in suction behind the injection, in the bore, creating a continuous fluid motion leading to a combined discharge of the two streams. Jet pumps typically consist of motive and secondary suction nozzles, throat and diffuser, as illustrated in Figures $1 \mathrm{a}$ and $1 \mathrm{~b}$. The relative position of the motive fluid and the suction nozzles with respect to the pump centreline determine the pump configuration as a central jet pump (CJP) or annular jet pump (AJP). In the CJP sketched in Fig.1a, the motive fluid is injected through the central nozzle and the secondary fluid drawn through the annular nozzle. In the AJP configuration in Fig. $1 \mathrm{~b}$, the fluids in the central and annular nozzles are reversed with respect to the CJP, resulting in an unobstructed flow path of the secondary fluid along the pump centreline [1].

A number of Reynolds-averaged Navier-Stokes (RANS) computational fluid 


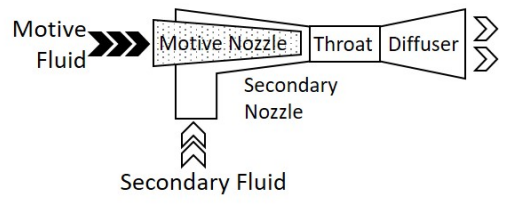

(a) Schematic of central jet pump.

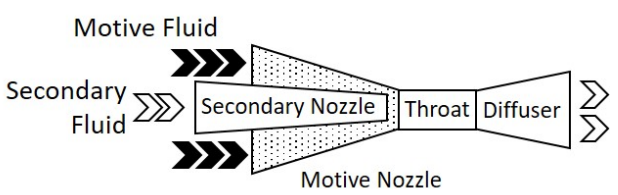

(b) Schematic of annular jet pump.

Figure 1: Jet pump configurations.

dynamics (CFD) studies have been conducted on the ability of turbulence models to capture the fluid flow phenomena of the turbulent flow through AJPs, with pressure, velocity and mass flow rates commonly used to quantify the performance of jet pumps. Kwon et al. [2] assessed the flow through a water-water AJP with 2D axisymmetric RANS simulations using the standard $k-\epsilon$ model [3] and the renormalisation group (RNG) $k-\epsilon$ model [4]. Both models gave predictions of the total pressure variations in the pump in good agreement with measured data but underpredicted the measured mass flow rate of the secondary stream, with the RNG estimates agreeing better with experimental mass flow rates. Further studies on a water-water AJP [5], comparing the predictions of the $k-\epsilon$ model to those of the standard Reynolds stress model (RSM) of Gibson and Launder [6], found that the $k-\epsilon$ model greatly overestimated the eddy viscosity. In turn, this resulted in excessive turbulent mixing and an underestimation of the pump performance. Conversely the RSM results correlated well with measured pressure and velocity, pointing to more accurate predictions of turbulence levels and structures. In a follow-up study of the same pump, using the same computational and experimental set-ups of [5], the motive fluid was induced with varying levels of swirl [7]. High levels of swirl intensity decreased the performance of the pump, whereas a low level increased the reported efficiency. As in [5], the $k-\epsilon$ model overpredicted turbulent mixing and underestimated the performance parameters in the pump, namely the flow rate of the secondary fluid and the pressure at the discharge. This mismatch increased with flow swirl. Conversely, the RSM results agreed well with experimental values, as in [5], even as the swirl intensity was increased. 
In some cases, RSM has also been found to predict marginally better agreement than linear eddy viscosity models for jet pumps without swirling flow. For example, Fan et al. $[8$ used the standard $k-\epsilon$, RNG $k-\epsilon$ and realizable $k-\epsilon$ models and RSM within ANSYS FLUENT ${ }^{\circledR} 2$ D axisymmetric simulations to assess the performance of a supersonic gas-gas CJP before carrying out its CFD-based design optimisation. RSM provided the best prediction of the measured curve of discharge pressure versus induced mass flow rate. The prediction improvement over the linear eddy viscosity models, however, was judged too small to justify the larger computational burden of using RSM in the design optimisation. Xu et al. 9] used Large Eddy Simulation (LES) CFD to study the flow field and the performance of a continuous jet water-water AJP. Numerical results agreed well with experimental measurements, both in terms of pressure fields and mass flow rates through the pump. Although no direct comparison of RANS and LES simulations of AJP swirling flows is yet available, it is plausible that LES analyses may resolve better than RANS analyses the complex flow physics of swirling AJP flows. It will have to be assessed whether the possibly better predictions achieved by using LES rather than RSM analyses outweighs the higher computational burden of the former method.

This study presents a fluid mechanic and performance analysis of a multinozzle AJP with no diffuser based on RANS CFD and experimental measurements carried out by the authors. The considered multi-nozzle AJP is a variant of the continuous annular jet pump in which the motive fluid is injected through multiple discrete nozzles distributed around the bore, as shown in Fig. 2, The motive fluid is compressed air, and the pumped fluid is air at ambient atmospheric conditions. The design of the multi-nozzle AJP allows the nozzles to be inclined both axially, by an angle $\alpha$, and circumferentially, by an angle $\beta$. The circumferential angle induces a swirling motion downstream of the injection point. Low levels of motive flow swirl have been shown to improve the efficiency of jet pumps by increasing fluid entrainment and suction [7, 10, 11, although the optimal level of swirl has yet to be determined for multi-nozzle AJPs. The CFD simulations of this study use both the $k-\omega$ Shear Stress 
Transport (SST) model [12 and RSM for the turbulence closure, and validation is based on comparisons of measured and computed static pressure fields at three operating regimes. Thorough cross-comparative investigations of this pump using both turbulence closures are presented with the aims of improving the understanding of its fluid mechanics, assessing and explaining the dependence of its performance on operating and design parameters, and assessing the predictive capabilities of the selected turbulence models for the analysis and future CFD-driven design optimisation of this device. To the best of the authors' knowledge, this report presents the first experimentally validated numerical study of multi-nozzle AJP flow physics and the impact of the choice of the RANS turbulence closure on its flow resolution.

The outline of the article is as follows: Section 2 presents the complete design of the considered multi-nozzle AJP, while Section 3 describes the AJP test rig. Section 4 defines physical domain, grids and boundary conditions of the CFD simulations and the selected CFD software and turbulence models. Thereafter, Section 5 analyses the sensitivity of the solution to the spatial and temporal refinement for each turbulence model. Section 6 starts by analysing the sensitivity of the AJP static pressure field and performance to the compressed air total pressure making use of both CFD results and experimental data. This is followed by a detailed cross-comparative analysis of the AJP flow physics based on both the SST and RSM simulations. Thereafter, the sensitivity of the pump flow field and performance to key design parameters is analysed to provide further design guidelines. A summary of the main findings is provided in Section 7 .

\section{Multi-nozzle jet pump}

As shown in the schematic of Fig. 2, the considered AJP consists of four parts, namely motive inlet pipe, annular plenum chamber, motive nozzles and pump bore. High-pressure air is stored in the plenum chamber, and is converted into kinetic energy in the nozzles that inject high-speed jets into the pump bore. 
The six nozzles of the pump are cylindrical with a diameter of $2 \mathrm{~mm}$, and the pump bore has diameter of $50 \mathrm{~mm}$. The nozzles of the pump connect to the pipe bore at an axial angle $\alpha$ of $35^{\circ}$ and a circumferential angle $\beta$ of $20^{\circ}$.
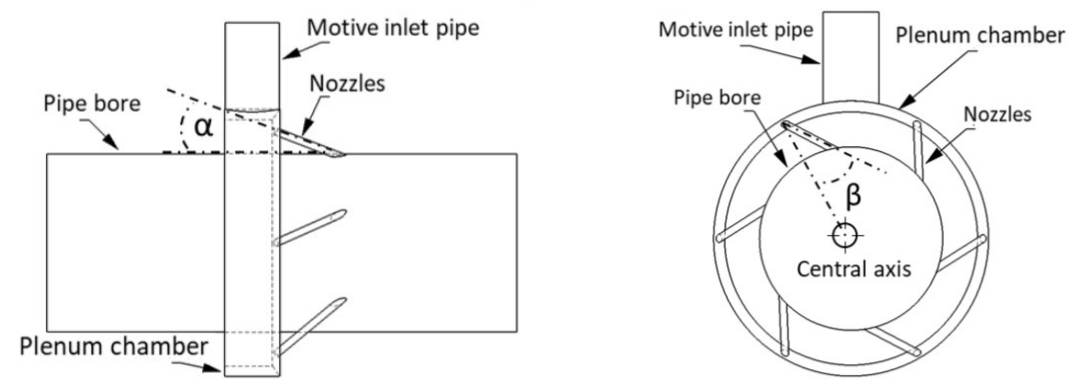

Figure 2: Multi-nozzle annular jet pump.

\section{Test rig}

A schematic of the test rig is shown in Fig. 3. The rig consists of the multinozzle AJP connected to a 1/2"-diameter compressed air line and two $50 \mathrm{~mm}$ diameter pipes, namely pipe $\mathrm{X}$ on the suction side and pipe $\mathrm{Y}$ on the discharge side, as reported in Fig. 3. The figure also indicates the components used to control and measure the compressed air flow, which are a throttle valve to adjust the pressure of the motive air and a digital pressure gauge with accuracy of \pm 0.05 bar before the pump. In the analyses below, the gauge total pressure of the compressed air in the pump annular plenum chamber is taken to be the value indicated by this pressure gauge.

Flush mounted pressure taps are fitted onto pipe $\mathrm{X}$ and $\mathrm{Y}$ to measure and record the wall static pressure in these ducts. Pipe $\mathrm{X}$ has 12 pressure taps distributed in the axial direction, whereas pipe $\mathrm{Y}$ has 19 pressure taps distributed in both the axial and the circumferential directions. In Pipe Y, there are 8 axial stations. The first three stations after the pump have four holes $90^{\circ}$ apart, with three of these used for pressure taps, and one used for inserting a Pitot tube. The remaining five axial stations feature two pressure taps $180^{\circ}$ apart, and a 
hole for the Pitot tube placed $90^{\circ}$ in between. Pitot tubes inserted in pipes $\mathrm{X}$ and $\mathrm{Y}$ through lateral holes with pressure head positioned on the centreline of the pipes, are used to measure and record the time-averaged static pressure at this location. Pressure readings on the pressure taps and the Pitot tubes are taken using a Kane 3200 differential pressure meter, which has a range of $\pm 9999 \mathrm{~Pa}$ and a resolution of $1 \mathrm{~Pa}$. The digital readings are verified with a fluid column multi-manometer to ensure the digital meter is accurately calibrated.

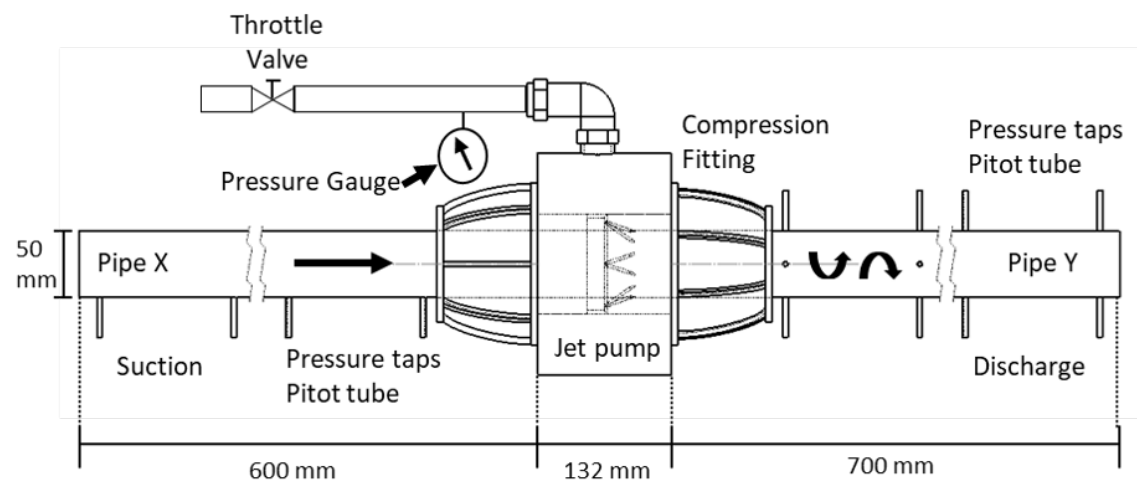

Figure 3: Experimental set-up.

\section{Numerical set-up}

\subsection{Physical domain, operating conditions and computational grids}

The physical domain, surface details of one of the adopted CFD meshes, and the selected far field boundary conditions (BCs) are shown in Fig. 4. All grids have been generated using ANSYS Meshing, and consist of multiple sub-domains featuring either hexahedral elements (structured sub-domains) or tetrahedral elements (unstructured sub-domains). A structured sub-domain is used in the initial part of the suction pipe and the final part of the discharge pipe. The axial extents of the structured sub-domains, the portions of the unstructured sub-domains before and after the plenum chamber, and the plenum chamber are quoted in Fig. 4, which also reports the main pipe inner diameter and the plenum chamber outer diameter. 


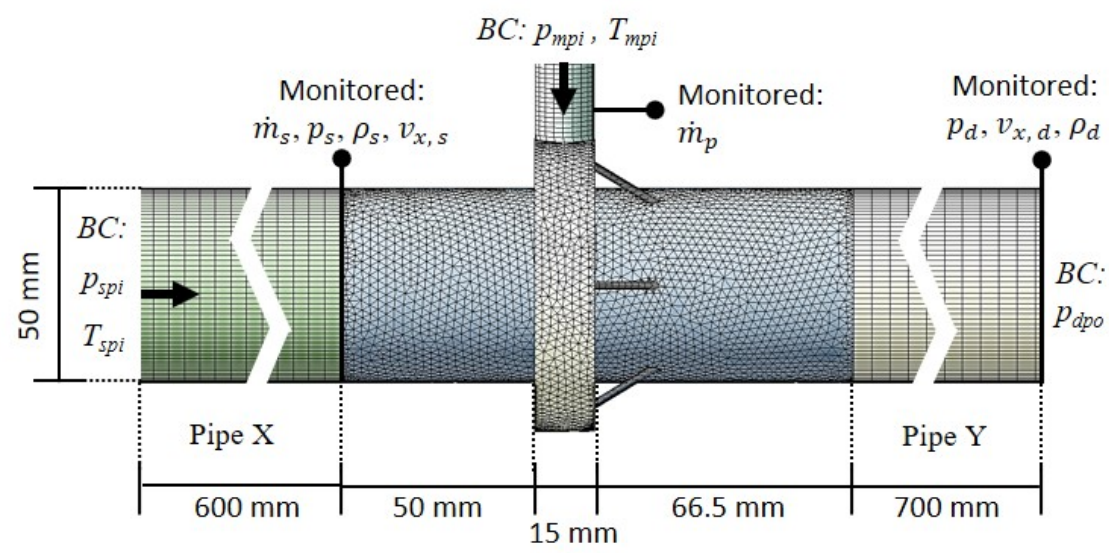

Figure 4: Physical domain and surface grid view.

Four levels of mesh refinement have been considered, corresponding to the grid parameters provided in Tab. 1. The number $N_{\text {circ }}$ of circumferential nodes in the suction and discharge ducts, and the number $N_{\text {axial }}$ of axial nodes from the inlet section of the suction duct to the outlet section of the discharge duct are reported in the second and third columns of the table, respectively. The number $N$ of elements of the adopted grids varies from $6.25 \mathrm{M}$ to $50 \mathrm{M}$. All boundary layers have been resolved by ensuring an adequate cell count through inflation layering along all walls, and selecting a minimum wall distance of the first grid nodes off viscous walls from the walls themselves yielding a mean nondimensionalised minimum wall distance $y^{+}$of order 1 , as reported in the fifth column of Tab. 1. Both steady and time-dependent simulations have been performed in this study. The value of the time step $\Delta t$ used for the timedependent simulation on each of the four spatial grids is provided in the sixth column of the table. 


\begin{tabular}{||llllll||}
\hline Grid & $N_{\text {circ }}$ & $N_{\text {axial }}$ & $N$ & Mean $y^{+}$ & $\Delta t[\mathrm{~s}])$ \\
\hline \hline Coarse & 71 & 1239 & $6.25 \mathrm{M}$ & 0.612 & $10^{-2}$ \\
\hline Medium & 100 & 1752 & $12.5 \mathrm{M}$ & 0.477 & $10^{-3}$ \\
\hline Fine & 150 & 2478 & $25 \mathrm{M}$ & 0.451 & $10^{-4}$ \\
\hline Extra-fine & 200 & 3504 & $50 \mathrm{M}$ & 0.424 & $10^{-5}$ \\
\hline
\end{tabular}

Table 1: Spatial and temporal parameters of selected four grid levels.

All flow simulations have used the compressible flow model. Gauge total pressure $p_{s p i}$ and total temperature $T_{s p i}$ are enforced at the inlet of the suction pipe. The reference pressure $p_{\text {ref }}$ is set to $101,325 \mathrm{~Pa}$, a value representative of the ambient pressure during the experiments. As the absolute total pressure at the suction duct inlet is taken to equal the ambient pressure, one has $p_{s p i}=0$ bar. The value of $T_{s p i}$ is $300 \mathrm{~K}$, also representative of the experimental conditions. Gauge total pressure $p_{m p i}$ and total temperature $T_{m p i}$ are also enforced at the inlet of the compressed air pipe. The pressure $p_{m p i}$ is set to the value of the total pressure of the compressed air after the flow meter shown in Fig. 3 and the total temperature $T_{m p i}$ is set to the ambient temperature of $300 \mathrm{~K}$. The pump fluid dynamics has been analysed for three values of $p_{m p i}$, namely 0.5, 1.0 and 2.0 bar, in differential pressure form. A static pressure outlet BC is applied at the outlet of the discharge pipe, where the gauge static pressure $p_{d p o}$ on the duct centreline is set to the value measured in the experiments. At this boundary, the static pressure is assumed to be circumferentially uniform but varying radially, and the value at each radius is determined by means of a radial equilibrium condition. The Reynolds number considered in this study is computed using a constant kinematic viscosity of $1.7894 \cdot 10^{-5} \mathrm{~m}^{2} / \mathrm{s}$, a velocity of $33 \mathrm{~m} / \mathrm{s}$ at the suction duct inlet, obtained using experimental estimates of the centreline dynamic pressure for the operating condition with $p_{m p i}=2.0 \mathrm{bar}$, and the main pipe diameter of $50 \mathrm{~mm}$. Adopting these values gives a Reynolds number of about $92 \mathrm{~K}$.

Some of the flow variables and integral outputs monitored in the simulations 
are indicated in Fig. 4. They include the air mass flow rate in the suction pipe $\dot{m}_{s}$, the mass flow rate of the compressed air $\dot{m}_{p}$ entering the pump bore, and the area-averaged values of static pressure $p_{s}$, density $\rho_{s}$ and axial velocity $v_{s, x}$ in the suction duct, and static pressure $p_{d}$, density $\rho_{d}$ and axial velocity $v_{d, x}$ at the pump discharge.

\section{2. $C F D$ code}

The finite volume CFD code ANSYS Fluent release 19.1 is used to simulate the flow field in the computational domain containing the multi-nozzle AJP making use of the operating conditions specified above. Simulations use a single-phase ideal gas compressible flow model. The compressible model is selected to account for the compressible flow effects associated with the large velocity variations due to high-speed nozzle jets at Mach close to 1, decelerating to the overall low speeds of the secondary flow in the range of Mach 0.1. The spatial discretisation of the governing equations is second order accurate and uses the upwind scheme. The pressure-based COUPLED solver, whereby the momentum and continuity equations are integrated in a strongly coupled fashion is used for integrating the steady flow equations, and also the timedependent equations at each physical time of unsteady flow simulations. A dual-time-stepping approach is used to solve time-dependent problems, and the discretisation of the physical time-derivatives is also second order accurate in time. The effects of turbulence are considered by solving the Reynolds-averaged Navier-Stokes form of the governing equations, and both an eddy viscosity model and a Reynolds stress model are used and compared for the turbulence closure.

\subsection{Turbulence modelling}

The $k-\omega$ shear stress transport model [12] is a two-equation eddy viscosity model that combines the $k-\epsilon$ and $k-\omega$ models, benefiting from the low sensitivity to the far field value of the turbulence dissipation rate $\epsilon$ of the former model and the good resolution of wall boundary layers of the latter model, even in the presence of adverse pressure gradients and moderate flow separation. 
Similarly to the $k-\omega$ and $k-\epsilon$ models, the SST model assumes an isotropic eddy viscosity, and employs Boussinesq approximation to estimate the Reynolds stress tensor. In the SST model, the turbulent viscosity $\mu_{t}$ is given by $\mu_{t}=$ $a_{1} \rho k / \max \left(a_{1} \omega, \Omega F_{2}\right)$, where $\rho, k$ and $\omega$ are respectively fluid density, turbulent kinetic energy (TKE) and specific dissipation rate per unit mass, $\Omega$ denotes the magnitude of the flow vorticity and $a_{1}=0.31$. The variable $F_{2}$ is a blending function that depends on $k, \omega$, the molecular viscosity $\mu$ and the distance to the nearest wall, and limits the transport of turbulent shear stress in highvorticity regions. The local values of $k$ and $\omega$ are determined by solving two transport equations including convection, diffusion and source terms. These two equations are coupled to the RANS equations due to the dependence of the turbulence model on the mean flow velocity and its gradient. The incompressible formulation of the transport equations for $k$ and $\omega$ are given by Eq. (1) and Eq. (2), respectively.

$$
\begin{gathered}
\frac{\partial}{\partial t}(\rho k)+\frac{\partial}{\partial x_{i}}\left(\rho k U_{i}\right)=\frac{\partial}{\partial x_{j}}\left(\mu+\frac{\mu_{t}}{\sigma_{k}} \frac{\partial k}{\partial x_{j}}\right)+G_{k}-Y_{k} \\
\frac{\partial}{\partial t}(\rho \omega)+\frac{\partial}{\partial x_{i}}\left(\rho \omega U_{i}\right)=\frac{\partial}{\partial x_{j}}\left(\mu+\frac{\mu_{t}}{\sigma_{\omega}} \frac{\partial \omega}{\partial x_{j}}\right)+G_{\omega}-Y_{\omega}+D_{\omega}
\end{gathered}
$$

in which $u_{i}$ denotes the $i^{\text {th }}$ component of the flow velocity vector. The symbols $\sigma_{k}$ and $\sigma_{\omega}$ are the turbulent Prandtl numbers of $k$ and $\omega$, respectively, and they belong to the set of constants of the turbulence model. The source terms $G_{k}$ and $Y_{k}$ in Eq. (1) denote respectively the production and destruction terms of $k$. Similarly, the source terms $G_{\omega}$ and $Y_{\omega}$ in Eq. 2 denote the production and destruction terms of $\omega$. The transport equation of $\omega$ contains an additional source term, the so-called cross-diffusion term $D_{\omega}$, which depends on the product of the gradients of $k$ and $\omega$. The definition of all five source terms can be found in 12 and $[13$.

The Reynolds stress model is an alternative RANS turbulence modelling approach that, although computationally more expensive than two-equation eddy viscosity turbulence models, has been shown to provide more reliable predic- 
tions than the latter models in the analysis of swirling jet pump flows [7, 14, due to its ability to better resolve streamline curvature and swirling flow effects, and the resulting changes in strain rate [15, 16, 17. The Boussinesq hypothesis of the $k-\omega$ SST model is not used in RSM, which instead solves a transport equation for each of the six distinct entries $\tau_{i j}=\overline{u_{i}^{\prime} u_{j}^{\prime}}$ of the Reynolds stress tensor, obtained by Reynolds-averaging the Navier-Stokes equations. Modelling directly each Reynolds stress, thus abandoning the assumption of isotropic eddy viscosity, enables RSM to partly account for the anisotropic character of turbulence in complex flows like that analysed in this study. The transport equation for each Reynolds stress component $\tau_{i j}$ is:

$$
\frac{\partial}{\partial t} \rho \tau_{i j}+\frac{\partial}{\partial x_{k}}\left(\rho U_{k} \tau_{i j}\right)=D_{T, i j}+D_{L, i j}+P_{i j}-\epsilon_{i j}+\phi_{i j}
$$

in which $D_{L, i j}$ and $D_{T, i j}$ are respectively the molecular and (modelled) turbulent diffusive terms, whose expressions are:

$$
D_{L, i j}=\frac{\partial}{\partial x_{k}}\left(\mu \frac{\partial \tau_{i j}}{\partial x_{k}}\right) \quad D_{T, i j}=\frac{\partial}{\partial x_{k}}\left(\frac{\mu_{t}}{\sigma_{k}} \frac{\partial \tau_{i j}}{\partial x_{k}}\right)
$$

The symbol $P_{i j}$ denotes the source term defining the production of $\tau_{i j}$ [18] and is given by:

$$
P_{i j}=-\rho\left[\tau_{i k} \frac{\partial U_{j}}{\partial x_{k}}+\tau_{j k} \frac{\partial U_{i}}{\partial x_{k}}\right]
$$

whereas $\epsilon_{i j}$ is a modelled source term controlling the dissipation of $\tau_{i j}$. Different choices are available in the literature to estimate this term. In general the calculation of $\epsilon_{i j}$ requires solving an additional transport equation, which, in this study, was chosen to be the $\omega$ equation of Menter's $k-\omega$ baseline (BSL) model [12, which is the same $\omega$ equation used in the SST model, and is thus Eq. (2). Using the value of $\omega$ obtained by solving Eq. (2), the dissipation rate is obtained using the equation:

$$
\varepsilon_{i j}=\frac{2}{3} \delta_{i j} \rho \beta_{R S M}^{*} k \omega
$$

where $\delta_{i j}$ is the Kronecker delta function, and the TKE, $k$, is obtained from the trace of the Reynolds stress tensor. The value of $\omega$ obtained from Eq. (2) 
is also used to compute the turbulent viscosity in the expression of $D_{T, i j}$, using the relation $\mu_{t}=\rho C_{\mu} \frac{k}{\omega}$, where $C_{\mu}=0.09$ [13].

The symbol $\phi_{i j}$ denotes the pressure strain source term, which describes the energy redistribution among the Reynolds stresses, the interaction of the mean velocity gradient field, and the fluctuating velocity field. In RSM, this source term is modelled as:

$$
\begin{aligned}
\phi_{i j}= & -C_{1} \rho \beta_{R S M}^{*} \omega\left[\overline{u_{i}^{\prime} u_{j}^{\prime}}-2 / 3 \delta_{i j} k\right]-\hat{\alpha}_{0}\left[P_{i j}-1 / 3 P_{k k} \delta_{i j}\right] \\
& -\hat{\beta}_{0}\left[D_{i j}-1 / 3 P_{k k} \delta_{i j}\right]-k \hat{\gamma}_{o}\left[S_{i j}-1 / 3 S_{k k} \delta_{i j}\right]
\end{aligned}
$$

where

$$
D_{i j}=-\rho\left[\overline{u_{i}^{\prime} u_{m}^{\prime}} \frac{\partial U_{m}}{\partial x_{j}}+\overline{u_{j}^{\prime} u_{m}^{\prime}} \frac{\partial U_{m}}{\partial x_{i}}\right]
$$

and $S_{i j}$ is the component $(i, j)$ of the mean strain rate, given by

$$
S_{i j}=\frac{1}{2}\left(\frac{\partial U_{j}}{\partial x_{i}}+\frac{\partial U_{i}}{\partial x_{j}}\right)
$$

It is thus seen that RSM requires the solution of seven additional transport equations, whereas the $k-\omega$ SST model requires the solution of only two additional equations. The choice of the $\omega$ equation of the $k-\omega$ SST and BSL models for calculating $\epsilon_{i j}$ in the RSM framework, makes the BSL-RSM advantageous over $\epsilon$-based RSM, as the former variant does not require any alteration of the equation for the dissipation rate near viscous walls to obtain accurate near-wall flow predictions.

\section{Mesh sensitivity analyses}

This section presents a sensitivy study of the $k-\omega$ SST simulations (Subsection 5.1 and RSM simulations (Subsection 5.2 of the multi-nozzle AJP flow field to both the spatial and the temporal refinement of the CFD amalysis. The simulations refer to a compressed air gauge pressure $p_{m p i}=2.0 \mathrm{bar}$, the largest of the three values considered in this research and that for which the flow complexity is expected to be highest.

Four levels of spatial and temporal refinement have been considered, with the key spatial and temporal grid parameters provided in Tab. 1. The $k-\omega$ SST 
steady simulations have been run with a number of iterations varying from 5, 000 on the coarse level to 25,000 on the finest grid, and the RSM simulations with this number varying from 7,500 on the coarse level to 25,000 on the finest grid. These values have ensured a residual reduction of four orders of magnitude in all cases. Each unsteady simulation has been initialised using the corresponding steady state solution, and the number of iterations per time-step has been set to 30 in all cases. This set-up has resulted in a residual reduction of three orders of magnitude at all physical times.

Both the $k-\omega$ SST and RSM mesh sensitivity studies below are carried out at two levels. At the detailed flow level, the axial profiles of the static pressure at the duct wall and on the duct centreline from the inlet of the suction duct to the pump discharge computed with all four grids are cross-compared. For validation purposes, these profiles are also compared to their measured counterparts. At an integral output level, the sets of steady and time-dependent SST and RSM simulations of the four grids are also cross-compared using two performance metrics of the multi-nozzle AJP, namely the pump power $P_{\text {out }}$ and efficiency $\eta$. The performance metric $P_{\text {out }}$ is the net power increment of the secondary flow power due to the energy provided by the jet system. Its definition is:

$$
P_{\text {out }}=\dot{m}_{s}\left[\left(\frac{p_{d}}{\rho_{m}}+\frac{v_{d, x}^{2}}{2}\right)-\left(\frac{p_{s}}{\rho_{m}}+\frac{v_{s, x}^{2}}{2}\right)\right]
$$

where, as explained in Section 4.1 and highlighted in Fig. 4. $\dot{m}_{s}, p_{s}$ and $v_{s, x}$ denote respectively mass flow rate, and area-averaged differential static pressure and axial velocity component of the air in the suction duct before encountering the compressed air jets, $p_{d}$ and $v_{d, x}$ denote respectively the area-averaged differential static pressure and axial velocity component at the discharge section of the pump, and $\rho_{m}$ is the mean of the area-averaged density $\rho_{s}$ in the suction duct and the area-averaged density $\rho_{d}$ the pump discharge. The expression of $P_{\text {out }}$ relies on the incompressible flow definition of the total pressure. This choice is justified by the fact that the fluid velocities at the position where the quantities in Eq. (6) are computed, are well below the compressibility threshold corresponding to Mach number 0.3. 
The definition of the pump efficiency $\eta$ is:

$$
\eta=\frac{P_{\text {out }}}{P_{\text {in }}}
$$

in which $P_{i n}$ is the pneumatic power injected into the system. Its definition is:

$$
P_{\text {in }}=\dot{m}_{p} \frac{p_{p}^{0}}{\rho_{p}^{0}}=\dot{m}_{p} R T^{0}
$$

where $\dot{m}_{p}$ is the overall mass flow rate of the jets. The symbols $p_{p}^{0}, \rho_{p}^{0}$ and $T_{p}^{0}$ denote the total pressure, total density and total temperature of the compressed air, and $R=287 \mathrm{~J} / \mathrm{Kg} / \mathrm{K}$. With reference to the notation of Fig. 4 . one has $p_{p}^{0}=$ $p_{\text {ref }}+p_{m p i}$, where $p_{\text {ref }}=101,325 \mathrm{~Pa}$ has been used, and $T_{p}^{0}=T_{m p i}=300 \mathrm{~K}$.

The performance metrics $P_{\text {out }}$ and $\eta$ also play a key role in the analysis of the sensitivity of some fluid dynamic features and the performance of the AJP to the compressed air pressure (Subsection 6.1), and the analysis of the fluid dynamic losses and the performance sensitivity to the nozzle diameter (Subsection 6.3.

\section{1. $k-\omega$ SST analyses}

The top plot of Fig. 5 compares the measured profile of the wall static pressure $p_{w}$ along the bore centreline between the inlet of the suction pipe and the discharge of the outlet pipe to the computed profiles of the steady SST simulations using the four grid levels with parameters summarised in Tab. 1. At each axial position, the numerical estimate of $p_{w}$ is obtained by circumferential averaging. In the experiment, time histories of the wall static pressure were measured only at the axial stations at which measured values are provided in Fig. 5 and at these stations the wall static pressure was measured at three circumferential positions at most. The provided measured values of $p_{w}$ are time and circumferential averages of these measurements, and the reported error bars correspond to the extreme values of the wall static pressure at each axial station. The bottom plot of Fig. 5 compares the measured profile of the static pressure $p_{x}$ on the bore centreline between the inlet of the suction pipe and the discharge of the outlet pipe to the four computed profiles of the steady SST simulations. At each axial station, the experimetal values of $p_{x}$ are the 
averages of time measurements carried out using a Pitot tube. No error bars are provided for the profile of $p_{x}$ because the time fluctuations of this variable are very small. The axial position $X$ along the horizontal axes of both plots is measured from the inlet of the suction pipe where the total pressure $p_{s p i}$ is imposed. The computed pressure profiles of Fig. 5 highlight fairly small variations with the level of grid refinement. Close inspection of the computed profiles of $p_{w}$ and $p_{x}$ shows that the fine and extra-fine solutions are nearly superimposed, indicating that the fine grid is adequate for obtaining a fully gridindependent solution. The pressure profiles computed with the fine grid and the measured ones are in good agreement over the entire axial length of the physical domain. A particularly good agreement is observed between the fine grid and measured profiles of $p_{x}$, not only along the suction and outlet ducts, but also in the region of the jet injections. The agreement between measured and fine grid profiles of $p_{x}$ is also fairly good, except for a small overprediction of this pressure in the suction duct. The time-averaged profiles of $p_{x}$ and $p_{w}$ obtained with the four unsteady simulations using the time-steps $\Delta t$ in Tab. 1 are superimposed to their steady counterparts in Fig. 5, and are thus not reported for brevity. 


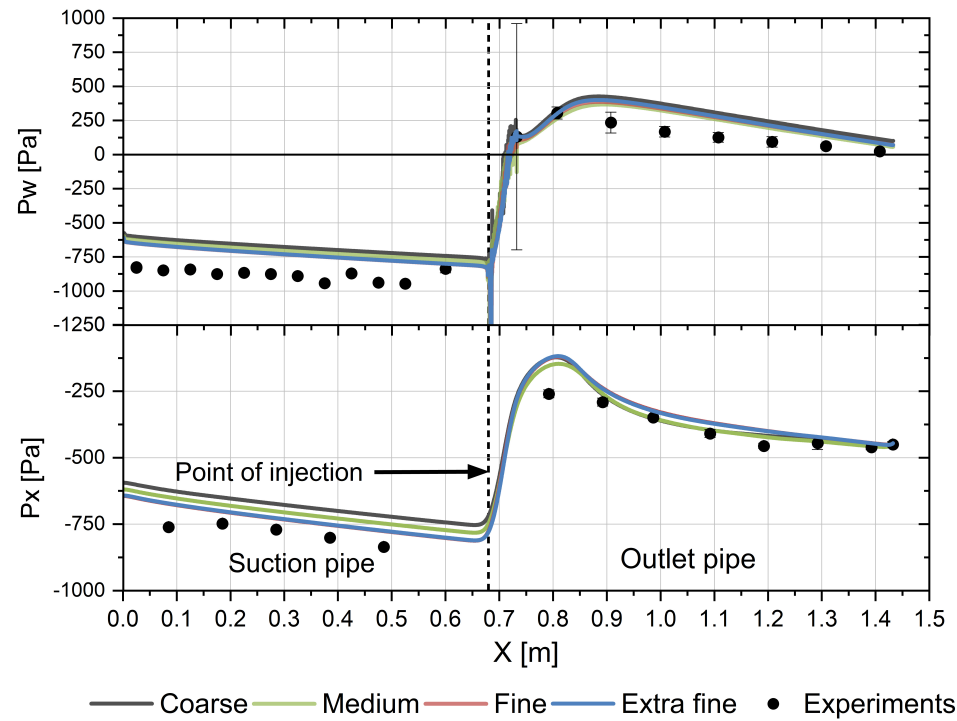

Figure 5: Axial profiles of duct static pressure obtained with experiments and steady $k-\omega \mathrm{SST}$ simulations on four grid levels: wall pressure $p_{w}$ (top), and centreline pressure $p_{x}$ (bottom).

The characteristic pressures, velocities and mass flow rates indicated in Fig.4 and the pump efficiency $\eta$ computed with the four steady and time-dependent simulations are presented in Tab. 2, whose second row provides the value of the time-step $\Delta t$ of the time-dependent simulations (no value is provided for the steady simulations). For both the steady and time-dependent analyses the variations of the monitored outputs between one grid level and the next finer one decrease notably passing from the coarse to the extra-fine grid, and gridindependent estimates of all outputs are obtained with the fine grid analysis for both analysis types. It is also found that the steady and time-dependent fine grid results differ very little from each other, confirming the suitability of the steady analysis for the flow and performance analyses presented below.

The values of the last row of Tab. 2 show that the considered pump has an efficiency of about $6 \%$, relatively low with respect to other jet pumps, which can achieve efficiencies of up to $40 \%$ [19]. This is because the considered AJP is a starting baseline, developed to investigate and clarify the main fluid dynamic 


\begin{tabular}{||l|l|l|l|l|l|l|l|l||}
\hline \hline & \multicolumn{2}{|c|}{ Coarse } & \multicolumn{2}{c|}{ Medium } & \multicolumn{2}{c|}{ Fine } & \multicolumn{2}{c|}{ Extra-fine } \\
\hline$\Delta t[\mathrm{~s}]$ & -- & $10^{-2}$ & -- & $10^{-3}$ & -- & $10^{-4}$ & -- & $10^{-5}$ \\
\hline \hline$p_{s}[\mathrm{~Pa}]$ & -741 & -741 & -772 & -771 & -801 & -802 & -800 & -802 \\
\hline$p_{d}[\mathrm{~Pa}]$ & -127 & -127 & -162 & -164 & -144 & -161 & -144 & -147 \\
\hline$v_{s, x}[\mathrm{~m} / \mathrm{s}]$ & 34.08 & 31.57 & 32.26 & 32.24 & 32.89 & 32.90 & 32.85 & 32.87 \\
\hline$v_{d, x}[\mathrm{~m} / \mathrm{s}]$ & 38.26 & 35.83 & 36.53 & 36.51 & 37.17 & 37.16 & 37.13 & 37.15 \\
\hline$\dot{m}_{s}[\mathrm{~kg} / \mathrm{s}]$ & 0.0726 & 0.0725 & 0.0741 & 0.0740 & 0.0755 & 0.0755 & 0.0754 & 0.0755 \\
\hline$\dot{m}_{p}[\mathrm{~kg} / \mathrm{s}]$ & 0.0104 & 0.0104 & 0.0104 & 0.0104 & 0.0104 & 0.0104 & 0.0104 & 0.0104 \\
\hline$\eta[\%]$ & 5.47 & 5.40 & 5.51 & 5.49 & 5.96 & 5.87 & 5.95 & 5.96 \\
\hline
\end{tabular}

Table 2: Pressures, velocities and mass flows computed with steady and time-dependent $k-\omega$ SST simulations on four grid levels (compressed air gauge pressure $p_{m p i}=2$ bar).

features enabling the motion of the secondary fluid. Moreover, there exist several different definitions of AJP efficiency, using which one may obtain different efficiency estimates for the same device. Indeed, the $P_{\text {out }}$ definition of Eq. (6) is rather conservative, in that it neglects the kinetic energy of the swirling flow at the pump discharge. This component has been deliberately omitted because it consists of energy that cannot be easily recovered in practical pumping applications. Although the optimisation of the device studies herein was beyond the scope of this research, the analyses in Subsection 6.3 investigate and discuss the reasons for the relatively low performance of this AJP, and propose guidelines to improve its design.

\subsection{Reynolds stress model analyses}

Only the coarse, medium and fine grid levels have been used to assess the sensitivity of the RSM simulations to the level of spatial and temporal refinement. The extra-fine grid level could not be used with RSM due to numerical instabilities preventing the achievement of adequate convergence levels. The top and bottom plots of Fig. 6 compare respectively the measured profile of the wall 
static pressure $p_{w}$ along the bore centreline and the measured profile of the static pressure $p_{x}$ on the bore centreline to the computed profiles of the steady RSM simulations using the three aforementioned grids. Similarly to the SST case, also the RSM wall and centreline pressure profiles reported in Fig. 6 highlight fairly small variations with the level of grid refinement. The medium and fine grid profiles of $p_{w}$ are in very good agreement along the entire duct length; the $p_{x}$ profiles computed with these two grids overlap along the discharge duct length region but present a reasonably constant small difference in the suction duct. It is noted that similar differences between the medium and fine grids have been also observed in the SST presure sensitivity analyses reported in Fig. 5 , which has shown that the fine grid solution is mesh independent. Also in the present RSM case, the pressure profiles computed with the fine grid and the measured ones are in good agreement over the entire axial length of the physical domain. The agreement between measured and fine grid profiles of $p_{x}$ appears to be even better than that obtained with the SST simulation, in that the fine grid RSM profile is closer to the measured profile in the suction duct. Similarly to the SST case, the time-averaged profiles of $p_{x}$ and $p_{w}$ obtained with the three unsteady simulations using the time-steps $\Delta t$ in Tab. 1 are superimposed to their steady counterparts in Fig. 5 , and are thus not reported for brevity. 


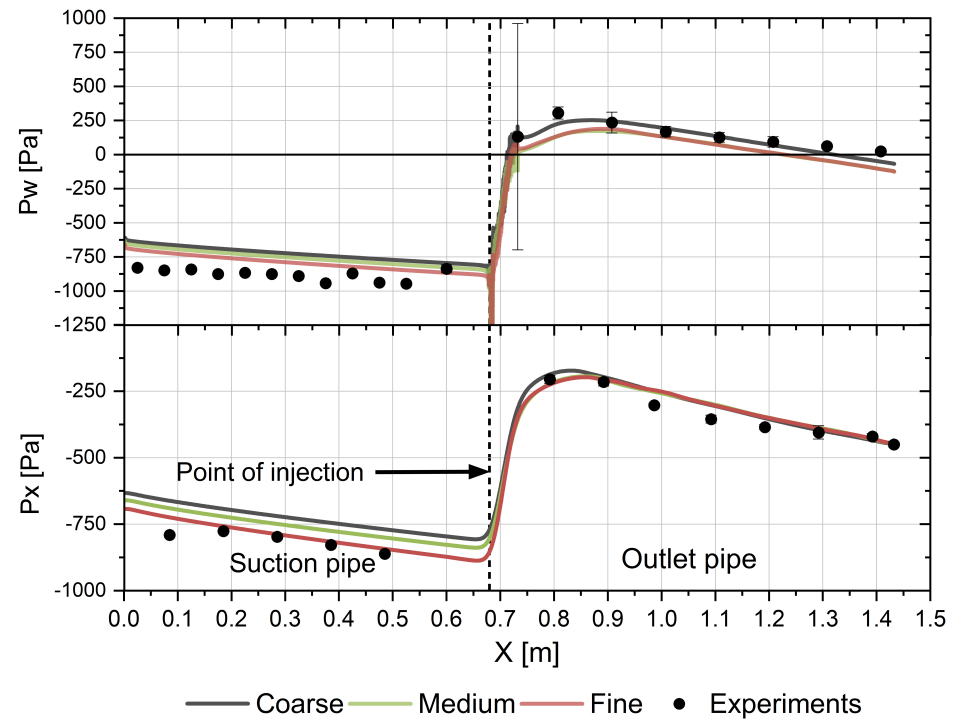

Figure 6: Axial profiles of duct static pressure obtained with experiments and steady RSM simulations on four grid levels: wall pressure $p_{w}$ (top), and centreline pressure $p_{x}$ (bottom).

The characteristic pressures, velocities and mass flow rates indicated in Fig.4 4 and the pump efficiency $\eta$ computed with the three steady and time-dependent simulations are presented in Tab. 3. The reported data highlight overall patterns of the aymptotic convergence to a grid-independent solution of both the steady and time-dependent RSM analyses that are similar to those of the SST mesh sensitivy analysis (Tab. 21, which concluded that the fine grid yields a gridindependent sollution. Using the fine grid RSM analysis, the pattern of the differences between the outputs of the steady simulation and the time-averaged output of the time-dependent simulations is similar to that of the fine grid SST analysis. These differences result in very small variations of the performance metrics of the pump. In light of the observed levels of sensitivity of the RSM solution to the spatial and temporal grid refinement, the fine grid has thus been used for all RSM analyses presented in the remainder of this report. 


\begin{tabular}{||l|l|l|l|l|l|l||}
\hline \hline & \multicolumn{2}{|c|}{ Coarse } & \multicolumn{2}{c|}{ Medium } & \multicolumn{2}{c||}{ Fine } \\
\hline$\Delta t[\mathrm{~s}]$ & -- & $10^{-2}$ & -- & $10^{-3}$ & -- & $10^{-4}$ \\
\hline \hline$p_{s}[\mathrm{~Pa}]$ & -797 & -794 & -828 & -817 & -870 & -856 \\
\hline$p_{d}[\mathrm{~Pa}]$ & -261 & -258 & -295 & -291 & -294 & -270 \\
\hline$v_{s, x}[\mathrm{~m} / \mathrm{s}]$ & 32.64 & 32.59 & 33.32 & 33.09 & 34.13 & 33.89 \\
\hline$v_{d, x}[\mathrm{~m} / \mathrm{s}]$ & 36.86 & 36.82 & 37.57 & 37.35 & 38.38 & 38.13 \\
\hline$\dot{m}_{s}[\mathrm{~kg} / \mathrm{s}]$ & 0.0749 & 0.0749 & 0.0765 & 0.0760 & 0.0783 & 0.0778 \\
\hline$\dot{m}_{p}[\mathrm{~kg} / \mathrm{s}]$ & 0.0103 & 0.0103 & 0.0103 & 0.0103 & 0.0103 & 0.0103 \\
\hline$\eta[\%]$ & 5.11 & 5.11 & 5.22 & 5.13 & 5.70 & 5.71 \\
\hline
\end{tabular}

Table 3: Pressures, velocities and mass flows computed with steady and time-dependent RSM simulations on three grid levels (compressed air gauge pressure $p_{m p i}=2$ bar).

\section{Results}

This section, consisting of three main parts, presents a thorough investigation of the flow field of the AJP that was experimentally tested in this project, and discusses the relationship between special fluid dynamic features of this device and its performance metrics. Making use of additional wall and centreline static pressure measurements taken while operating the device with compressed air gauge pressure $p_{m p i}$ of 1.0 and 0.5 bar and fine grid SST and RSM simulations of these regimes, Subsection 6.1 assesses the sensitivity of the flow physics resolution to the turbulence model choice, discusses the better suited selection of the latter, and analyses the dependence of the performance metrics on $p_{m p i}$. Subsection 6.2 presents a detailed comparative analysis of the AJP key fluid dynamic features for $p_{m p i}=2$ bar based on the fine grid RSM and SST simulations. These investigations are key to support the analyses and discussions of Subsection 6.3 on the effects of the velocity swirl induced by the jets. Subsection 6.3 also analyses additional efficiency loss causes, providing design-relevant information. 


\subsection{AJP pressure and performance sensitivity to compressed air pressure}

Measured SST and RSM axial profiles of $p_{w}$ and $p_{x}$ for the three operating conditions associated with values of the compressed air gauge total pressure $p_{m p i}$ of 2.0, 1.0 and 0.5 bar are provided in Figures $7 \mathrm{a}$ 7b and $7 \mathrm{c}$ respectively. Both sets of computed profiles have been obtained with the steady fine grid set-up, and the postprocessing steps required to obtain both the measured and computed profiles are the same as described in Subsection 5.1. Here, however, the circumferential variability of the computed wall static pressure at each axial position is also analysed. At each axial position of the computed $p_{w}$ profiles, the lower and upper bounds of the shaded area indicate the minimum and maximum values of the wall pressure in the circumferential direction. One notes that only the RSM simulations predict a significant level of circumferential variability of the wall static pressure after the jet injection, and that this variability decreases with $p_{m p i}$. This trend is qualitatively well captured by the RSM analyses, although the predicted variability is lower than that observed in the experiments.

The results of Fig. 7 show that both the SST and RSM pressure profiles are generally in good qualitative and quantitative agreement with the measured data. However, while both the $p_{w}$ and $p_{x}$ profiles predicted by the SST and RSM analyses are very close to each other for $p_{m p i}=0.5$ bar, the difference between the two variables obtained with the SST and RSM set-ups increases with $p_{m p i}$, and is thus maximum at $p_{m p i}=2$ bar. At this pressure, the RSM profile of the centreline static pressure $p_{x}$ before the compressed air injection points shows a lower level than the SST profile, indicating higher axial velocity and mass flow rate, in notably better agreement with the measured profile in this region. Shortly after the jet injection, the SST $p_{x}$ profile presents a more pronounced peak than the RSM profile, whose more gradual descent towards the static pressure value imposed on the centreline of the discharge section is more consistent with the experimental data. The difference between the RSM and SST profiles of wall static pressure $p_{w}$ is less pronounced than that of $p_{x}$, but also in this case the RSM estimate appears to be in better agreement with 


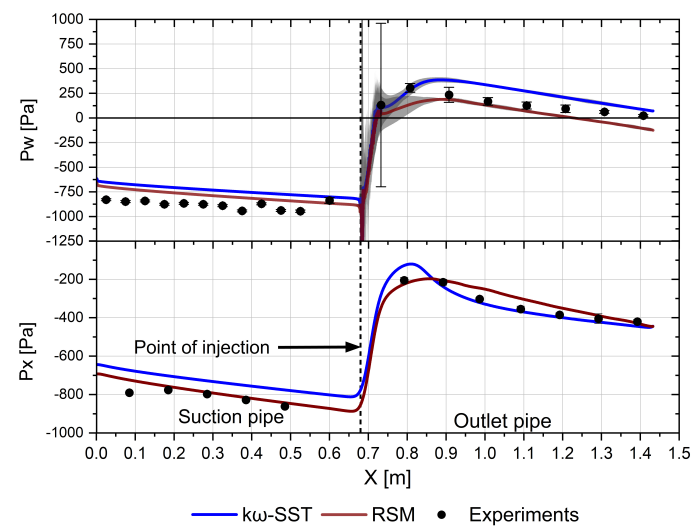

(a) $p_{m p i}=2.0$ bar

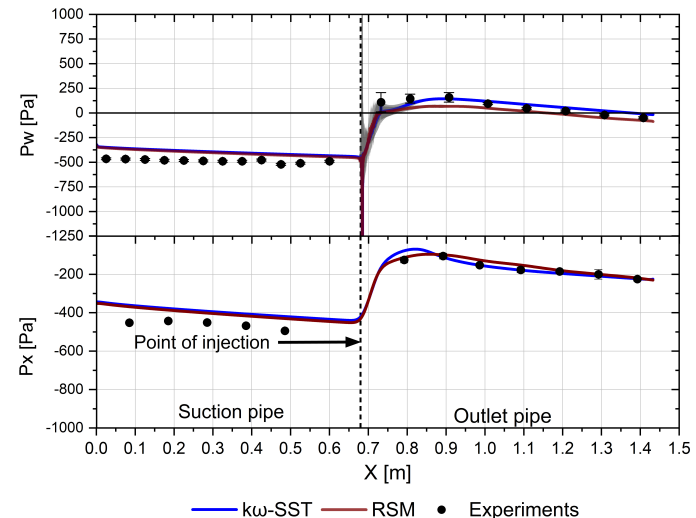

(b) $p_{m p i}=1.0$ bar

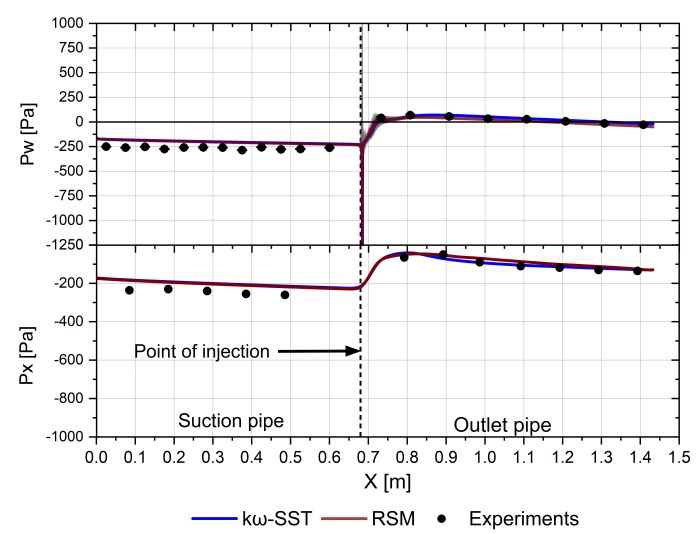

(c) $p_{m p i}=0.5$ bar

Figure 7: Axial profiles of wall $\left(p_{w}\right)$ and centreline $\left(p_{x}\right)$ static pressure obtained with experiments, and steady fine grid $k-\omega$ SST and RSM simulations for three values of the compressed air gauge pressure $p_{m p i}$. 
the experimental profile.

To investigate further the causes of the lower axial speed before the jets predicted by the $k-\omega \mathrm{SST}$ simulation of the operating condition at $p_{m p i}=2$ bar, and discuss the suitability of the two turbulence models to the analysis of all three operating conditions, it is instructive to introduce the swirl number $S_{n}$. This variable is the ratio of the mean axial flux of the angular momentum and the axial flux of linear momentum [20, and it provides a measure of the level of flow swirl. The expression of $S_{n}$ is:

$$
S_{n}=\frac{\int r v_{\theta} \vec{v} \cdot d \vec{A}}{R \int v_{x} \vec{v} \cdot d \vec{A}}
$$

where $R$ is the duct radius, $r$ denotes the radial distance from the duct centreline, and $\vec{v}, v_{\theta}$ and $v_{x}$ denote respectively the flow velocity vector, and the circumferential and axial components of $\vec{v}$.

The profiles of $S_{n}$ along the duct centreline for the three values of $p_{m p i}$ computed with the two simulation sets are reported in Fig. 8 In all cases, $S_{n}$ increases rapidly after the jet injection, and reaches a peak between 200 and $300 \mathrm{~mm}$ downstream of the injection point; the subsequent decay is predicted to be faster by the SST analyses. This indicates that after the peak, the tangential velocity increases at a lower rate than the axial velocity. The faster decay of $S_{n}$ predicted by the SST simulations is likely to be due to more effective mixing of the primary and secondary streams, a scenario supported by the conclusions of the cross-comparative analysis of the SST and RSM simulations of Subsection 6.2 From about $10 \mathrm{~cm}$ downstream of the injection point, the RSM analysis predicts a lower level of $S_{n}$ than the SST analysis for all three $p_{m p i}$ values, but the RSM swirl numbers between this position and the injection point for $p_{m p i}=1$ bar and, even more so, for $p_{m p i}=2$ bar, are higher than the SST $S_{n}$ values. From a physical viewpoint, it is expected that the jet velocity and the magnitude of the velocity swirl in the injection area increase with the total pressure of the compressed air. Numerically, the observations above highlight that as the strength of the swirling flow component increases, the RSM analysis tends to predict a higher swirl level in the jet region than the SST analysis. This 
results in overall larger velocity magnitudes and lower pressure in the injection region. This is the reason for lower static pressure in the suction duct predicted by the RSM analysis. In light of the very good agreement between the measured and RSM suction duct pressure profiles shown in Fig. 7a for the highest $p_{m p i}$ level, the swirl number analysis above provides further evidence supporting the better suitability of the RSM analysis for high swirl levels. It is also noted that the lower peak of $S_{n}$ of the RSM over the SST analysis is partly due to the lower axial momentum predicted by the latter simulation, an occurrence that increases the levels of $S_{n}$ even for constant angular momentum.

Swirling flows are often categorised in low- and high-swirl flows, and the transition threshold of the swirl number between the two classes is taken to be about 0.5 21. RSM is known to be better suited than two-equation eddy viscosity models to resolve flows characterised by high levels of swirl and streamline curvature 22. The RSM and SST $S_{n}$ curves reported in Fig. 8 show that the considered flow fields feature a medium level of swirl, as the maximum RSM value of this parameter is about 0.45 and the maximum SST value is about 0.55 , with the maximum values of both simulations being achieved for the highest value of the compressed air pressure. This occurrence is in line with the observation above based on the comparison of measured and predicted static pressure field in the pump bore that the RSM solution is closer to the experimental data than the SST solution at $p_{m p i}=2$ bar, whereas also the SST solution becomes rapidly closer to both the RSM solution and the experimental data when the compressed air pressure and, consequently, the flow swirl decreases. 


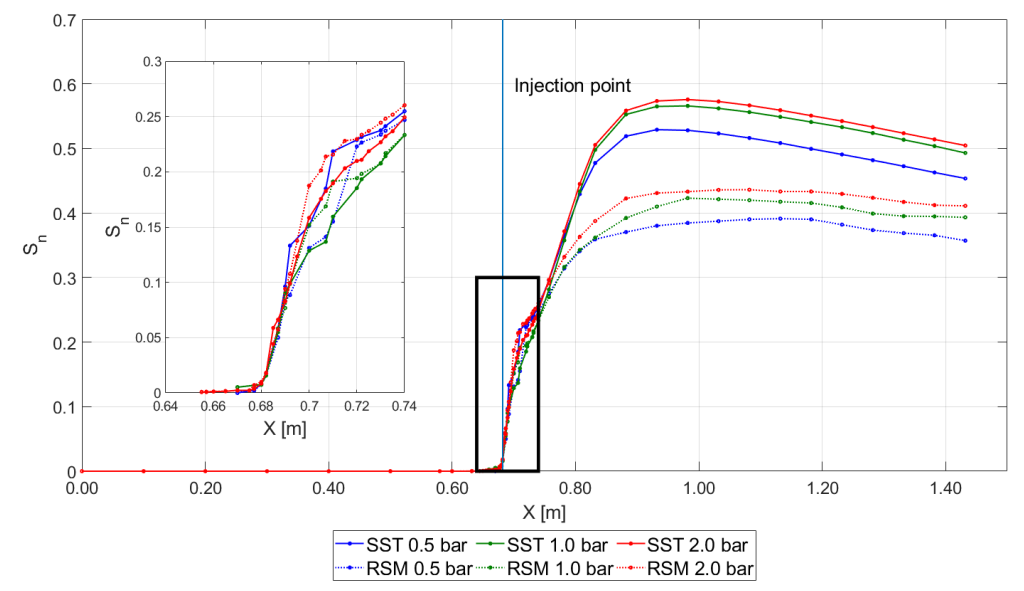

Figure 8: Swirl number $S_{n}$ along pump axis for three values of compressed air gauge pressure $p_{m p i}$.

A quantitative comparison of the pump performance predicted by the SST and RSM analyses for the three values of $p_{m p i}$ is presented in Tab. 4 The differences between all variables computed by the SST and RSM analyses increase with $p_{m p i}$, similarly to what is observed for the bore static pressure fields, and are maximum for $p_{m p i}=2$ bar. At this pressure, the RSM mass flow $\dot{m}_{s}$ in the suction duct is about $4 \%$ larger than the SST prediction, consistent with the lower duct centreline static pressure of the RSM simulation highlighted above. Conversely, the area averaged discharge pressure predicted by the RSM analysis is significantly lower that that predicted by the SST analysis, resulting in the former analysis predicting a lower total pressure rise, despite its predicted higher discharge kinetic energy. As the lower RSM total pressure rise outweighs the higher mass flow rate of secondary fluid in the calculation of the output power, the RSM estimate of $P_{\text {out }}$ is thus lower than the SST estimate. Also the RSM estimate of the pump efficiency is lower, as the input power predicted by the two analyses is comparable. It is also observed that both simulation set-ups predict a continuous increment of the pump efficiency with $p_{m p i}$, as the pneumatic power of the jets increases more slowly than the power imparted by 


\begin{tabular}{|l|l|l|l|l|l|l|}
\hline$p_{\text {mpi }}[\mathrm{bar}]$ & \multicolumn{2}{l}{0.5} & \multicolumn{1}{l|}{1.0} & 2.0 \\
\hline Model & $k-\omega \mathrm{SST}$ & RSM & $k-\omega \mathrm{SST}$ & RSM & $k-\omega \mathrm{SST}$ & RSM \\
\hline \hline$p_{s}[\mathrm{~Pa}]$ & -222 & -227 & -434 & -446 & -800 & -870 \\
\hline$p_{d}[\mathrm{~Pa}]$ & -65 & -89 & -103 & -162 & -144 & -294 \\
\hline$v_{s}, x[\mathrm{~m} / \mathrm{s}]$ & 16.97 & 17.10 & 23.98 & 24.26 & 32.85 & 34.13 \\
\hline$v_{d}, x[\mathrm{~m} / \mathrm{s}]$ & 19.05 & 19.17 & 26.89 & 27.17 & 37.13 & 38.38 \\
\hline$\dot{m}_{s}[\mathrm{~kg} / \mathrm{s}]$ & 0.0391 & 0.0394 & 0.0535 & 0.0558 & 0.0754 & 0.0783 \\
\hline$\dot{m}_{p}[\mathrm{~kg} / \mathrm{s}]$ & 0.0049 & 0.0049 & 0.0069 & 0.0068 & 0.0104 & 0.0103 \\
\hline$P_{\text {out }}[\mathrm{W}]$ & 6.69 & 6.11 & 19.03 & 17.08 & 53.58 & 50.56 \\
\hline$P_{\text {in }}[\mathrm{W}]$ & 419.9 & 418.4 & 598.3 & 590.0 & 900.1 & 887.5 \\
\hline$\eta \%$ & 1.59 & 1.46 & 3.18 & 3.00 & 5.95 & 5.70 \\
\hline
\end{tabular}

Table 4: Pressures, velocities, mass flows and performance metrics computed with steady fine grid $k-\omega \mathrm{SST}$ and RSM simulations for three values of compressed air gauge pressure $p_{m p i}$.

the jets to the secondary stream.

\subsection{Detailed fluid dynamic analyses}

This section presents detailed fluid dynamic analyses of the considered AJP working with a gauge total pressure $p_{m p i}$ of the compressed air of 2 bar based on a cross-comparative discussion of the steady fine grid SST and RSM simulations. The aim is to highlight the physics of the energy transfer, and assess qualitatively and quantitatively the differences between the predictions of the two turbulence model approaches for the problem type under consideration. 


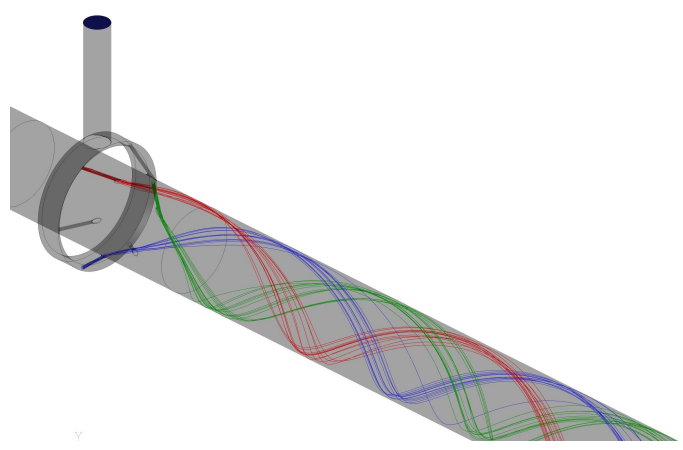

Figure 9: Swirling flow streamlines originating at jet injection computed by steady fine grid $k-\omega \mathrm{SST}$ analysis (compressed air gauge pressure $p_{m p i}=2$ bar).

The helical flow pattern associated with the jet system travelling downstream from the injection points is visualised in the streamline plot of Fig. 9, which refers to the SST solution. The plot reports the streamlines of three jets originating at three nozzles equally spaced in the circumferential direction. The inclination of the nozzles is such that the jets swirl in the clockwise direction when viewed from downstream. Fig. 9 highlights that, while remaining in the wall proximity as they travel downstream, the jet streamlines also undergo significant expansion, as indicated by their increasing divergence as they progress to the pipe exit.

To illustrate in greater detail how the jets of the motive fluid interact with the secondary fluid and the pipe wall as they travel downstream, the cross sectional contour plots of the velocity magnitude $|v|$ at six axial positions computed by the SST analysis are examined in Fig. 10. The six small red regions next to the nozzle exits in Fig. 10 -a are due to the high speed of the jets injected at this axial position. As the flow progresses to the axial position at $25 \mathrm{~mm}$ downstream of the injection points (Fig. $10 \mathrm{f}$ ), the area of the jet cross sections increases, due to their diffusion in the secondary fluid stream. It is also noted, as mentioned above, that the jets remain in the wall proximity from their injection to the $25 \mathrm{~mm}$ axial position, although from $15 \mathrm{~mm}$ (Fig. 10 d), a more significant growth in the radial direction is observed. From this position, one also notes the development of a wall boundary layer, corresponding to the dark blue low- 


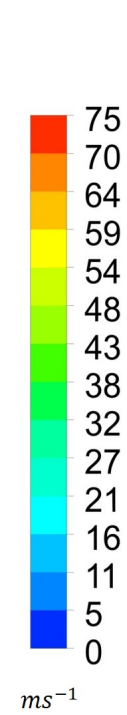

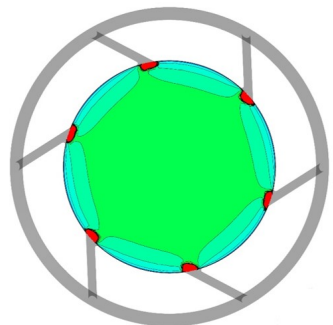

a) Injection

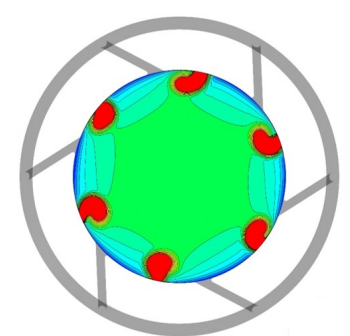

d) $15 \mathrm{~mm}$

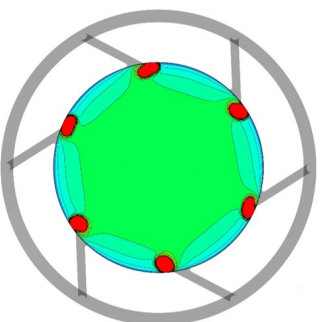

b) $5 \mathrm{~mm}$

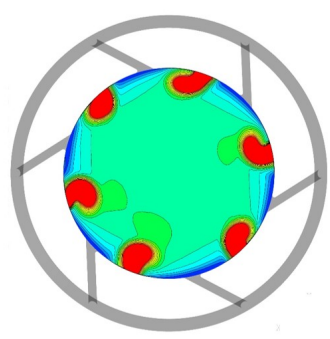

e) $20 \mathrm{~mm}$

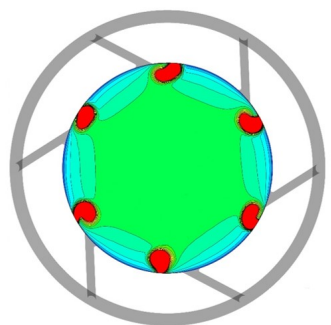

c) $10 \mathrm{~mm}$

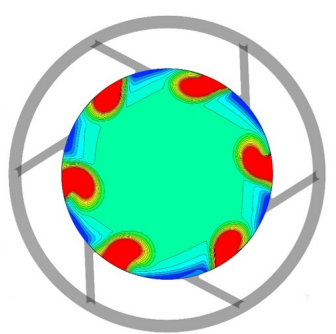

f) $25 \mathrm{~mm}$

Figure 10: Contours of velocity magnitude $|v|$ in axial sections after jet injection computed by steady fine grid $k-\omega \mathrm{SST}$ analysis (compressed air gauge pressure $p_{m p i}=2 \mathrm{bar}$ ).

speed strips between adjacent jets.

The development of the flow patterns before the injection points and from the $25 \mathrm{~mm}$ axial position is analysed in further detail by means of a cross comparison of the SST and RSM analyses in the subplots of Fig. 11. The subplots of this figure report cross sectional contour plots of velocity magnitude $|v|$, axial velocity $v_{x}$, and circumferential or swirl velocity $v_{\theta}$. Before the injection point (Fig. 11a), the velocity of the secondary flow is purely axial. At this axial station, the flow velocity contours predicted by the two turbulence models are comparable. At $25 \mathrm{~mm}$ after the injection (Fig. 11b), the SST and RSM jet cross sections feature overall similar patterns, highlighting moderate diffusion into the central part of the flow, more pronounced for the SST prediction. The SST velocity contours are closer to a cyclically symmetric pattern than the RSM contours. Further downstream, at $100 \mathrm{~mm}$ from the injection (Fig. 11c), the jets diffuse more into the secondary fluid stream, a process resulting in the compressed air jets increasing the axial momentum of the secondary fluid stream. 
At the $100 \mathrm{~mm}$ position, the overall flow progressively develops a predominantly axisymmetric pattern, as the circumferential non-uniformities become more attenuated. This is due to the jets energising the entire annulus boundary layer, as demonstrated by the fact that the low-speed near-wall patches between adjacent jets visible in Fig. 11b have disappeared in Fig. 11c, which instead shows a circumferentially more uniform high-speed flow near the wall. The near-wall fluid is seen to have higher axial velocity than the fluid in the central part of the pipe, due to the high energy of the jets. As indicated above, the swirling component of the flow does not penetrate all the way into the bore. This is demonstrated by both the SST and RSM $v_{\theta}$ contour plots of Fig. 11d. Comparing these two plots also shows that the $k-\omega$ SST model predicts a deeper penetration of the jets into the pipe bore. This is also illustrated in the meridional plane contour plots of the swirling velocity component discussed below.

The SST and RSM contours of the velocity magnitude $|v|$ in the bore meridional section containing the centreline of the compressed air pipe are reported in Figures $12 \mathrm{a}$ and $12 \mathrm{~b}$, respectively, whereas those of the axial velocity component $v_{x}$ are provided in Figures $12 \mathrm{c}$ and $12 \mathrm{~d}$, respectively, and those of the circumferential velocity component $v_{\theta}$ are provided in Figures $12 \mathrm{e}$ and $12 \mathrm{f}$, respectivley. All plots extend to an axial position about $400 \mathrm{~mm}$ downstream of the injection point. The $v_{x}$ contour plots of both simulations highlight the existence of two distinct regions after the jet injection: a high-axial speed annular region, and an inner lower-axial speed region. The $v_{\theta}$ contour plots also show that the aforementioned higher-speed annular region also has a high swirl whereas the velocity of the lower-speed core region is purely axial. This pattern of high axial and tangential velocity in the outer part of the bore after the jet injection has been observed and analysed in other swirling flows similar to those analysed in this report, including the numerical study of [23]. Downstream of the jet injection, viscous shear and turbulent mixing of the high- and low-speed regions result in a progressive acceleration of the central low-speed fluid flow. These trends are common to both SST and RSM predictions. However, the axial velocity of the central fluid stream predicted by the SST analysis is higher than 

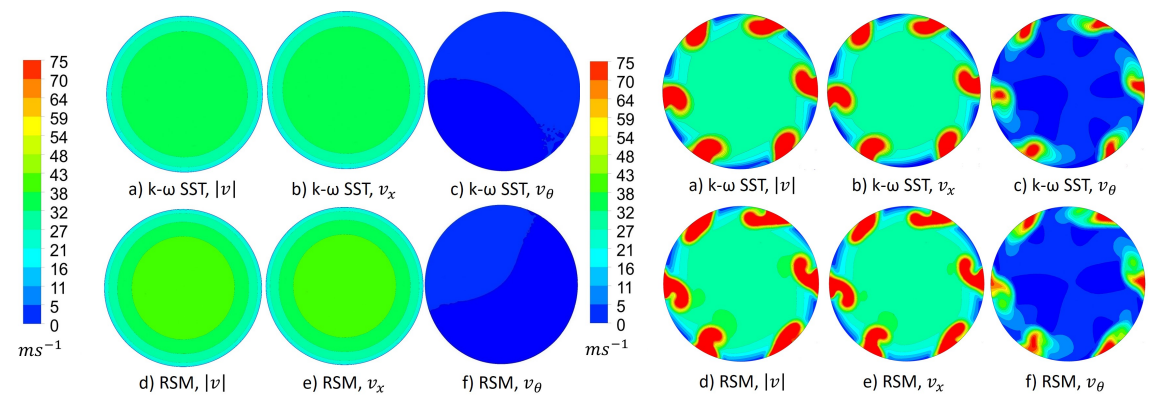

(a) $2.5 \mathrm{~mm}$ before jet injection

(b) $25 \mathrm{~mm}$ after jet injection

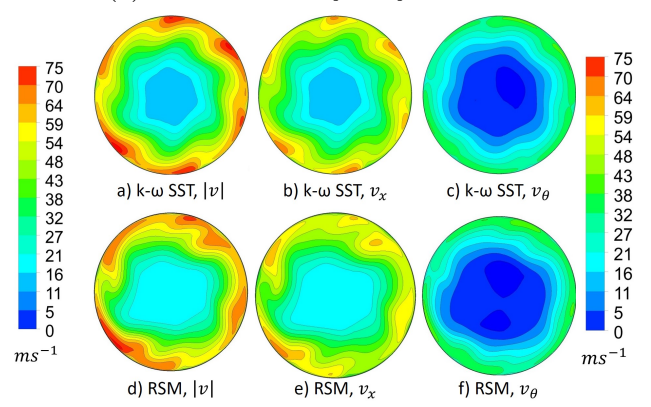

(c) $100 \mathrm{~mm}$ after jet injection

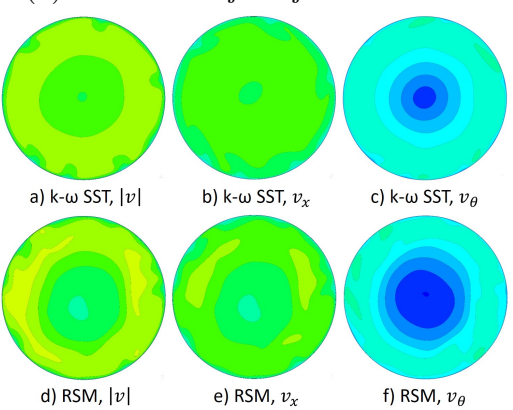

(d) $450 \mathrm{~mm}$ after jet injection

Figure 11: Contours of velocity magnitude $(|v|)$, and axial $\left(v_{x}\right)$ and circumferential $\left(v_{\theta}\right)$ velocity components in axial sections before and after jet injection computed by steady fine grid $k-\omega \mathrm{SST}$ and RSM analyses (compressed air gauge pressure $p_{m p i}=2$ bar). 
that predicted by the RSM analysis, whereas the conclusion is reversed with regard to the axial velocity of the higher-speed near-wall fluid stream. Crosscomparing the SST and RSM $v_{\theta}$ contour plots, one notes that the SST set-up predicts a deeper diffusion of the swirling stream into the pump bore than the RSM set-up does, pointing to a more effective mixing of motive and secondary fluids predicted by the SST analysis.

The eddy viscosity variable can be viewed as an index of the turbulent transfer of energy as a result of turbulent eddies [24, and it thus provides an estimate of the energy exchange between the two streams. Therefore it is instructive to inspect the SST and RSM eddy viscosity contour plots in the meridional plane provided in Fig. 13. The SST contours show that a high level of turbulent viscosity over the entire cross section of the duct is achieved closer to the injection point than in the RSM case, and that higher levels of turbulent viscosity in the bore central region are maintained until the discharge section. This and the observations made above in the analysis of the velocity contour plots further emphasise that the $k-\omega$ SST model predicts a higher level of penetration of the jets resulting in higher entrainment of secondary fluid. Therefore, it can be concluded the $k-\omega$ SST model predicts a higher level of mixing of primary and secondary fluid, which in turn leads to the higher level of predicted efficiency.

The static pressure contours obtained by both simulations at the pump discharge section are compared in Fig. 14. The static pressure level on the centreline is the same in both analysis, as expected, due to the use of a radial equilibrium pressure $\mathrm{BC}$ with given differential pressure of $-450 \mathrm{~Pa}$ on the centreline. However, a marked difference between the wall static pressure levels of the two simulations exist, which ultimately results in a significantly different radial profile of the static pressure at the exit station. In both simulations the wall static pressure increases fairly rapidly from the centreline to the wall, but such growth is faster for the SST radial profile, causing a wall pressure difference of $\approx 175 \mathrm{~Pa}$ between the two analyses. As a result, the SST area-average static pressure $p_{d}$ is higher than the RSM average. This is due to the SST anal- 


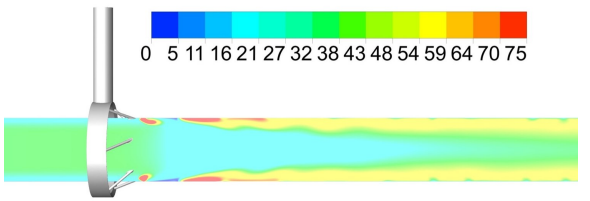

(a) $|v|, k-\omega \mathrm{SST}$

$m s^{-1}$

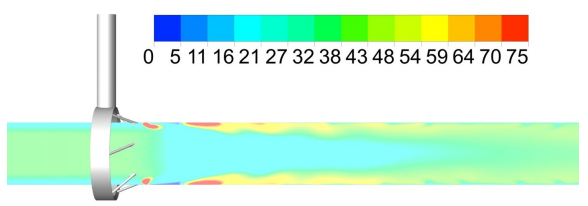

(c) $v_{x}, k-\omega \mathrm{SST}$

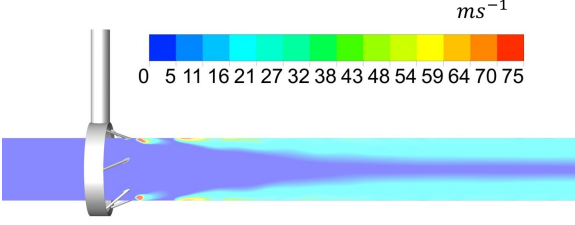

(e) $v_{\theta}, k-\omega \mathrm{SST}$

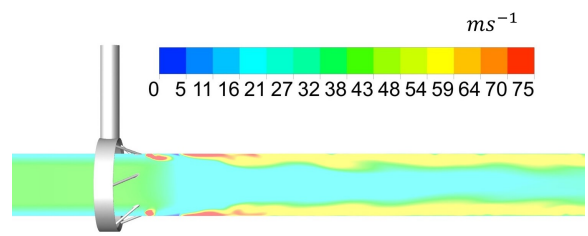

(b) $|v|$, RSM

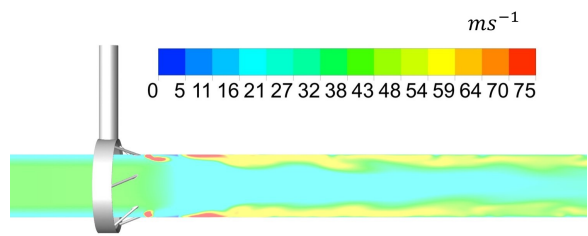

(d) $v_{x}, \mathrm{RSM}$

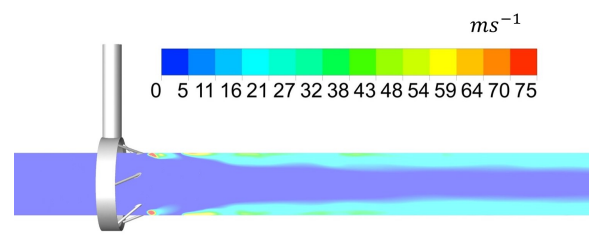

(f) $v_{\theta}, \mathrm{RSM}$

Figure 12: Contours of velocity magnitude $|v|$ (top), axial velocity component $v_{x}$ (middle) and circumferential velocity component $v_{\theta}$ in bore meridional section computed by steady fine grid $k-\omega$ SST and RSM analyses (compressed air gauge pressure $p_{m p i}=2$ bar).

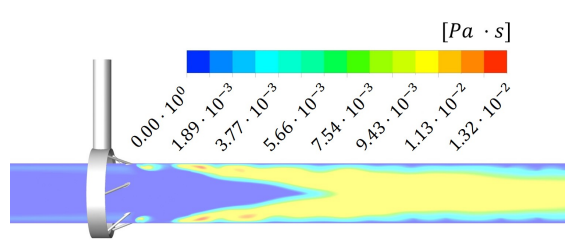

(a) $k-\omega \mathrm{SST}$

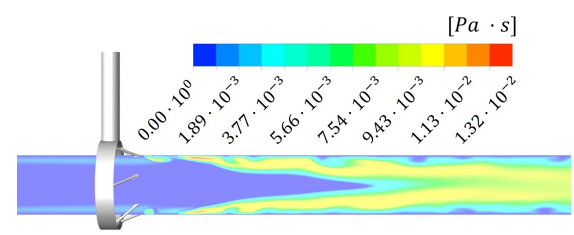

(b) RSM

Figure 13: Contours of eddy viscosity $\left(\mu_{t}\right)$ in bore meridional section computed by steady fine grid $k-\omega$ SST and RSM analyses (compressed air gauge pressure $p_{m p i}=2$ bar). 
ysis predicting a lower dissipation of the jet energy and a more effective mixing of jets and secondary stream. One of the effects of this lower dissipation is a higher level of exit total pressure predicted by the SST analysis, an occurrence highlighted in the SST and RSM total pressure contour plots at the discharge section shown in Fig. 15. It is noted that the existence of the so-called turbulent mixing region, in which the static pressure rises after the jet injection and an effective energy transfer between motive and secondary fluids due to jet mixing occurs has also been observed for water jet pumps [25].

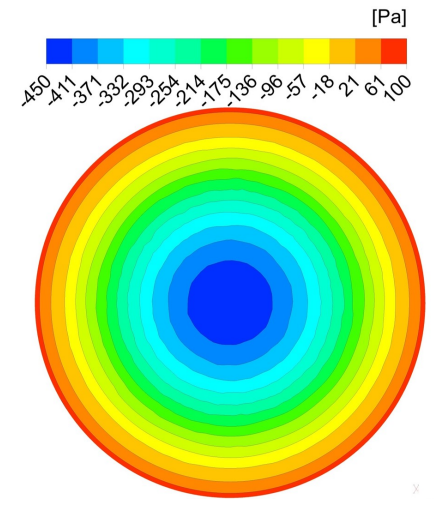

(a) $k-\omega \mathrm{SST}$

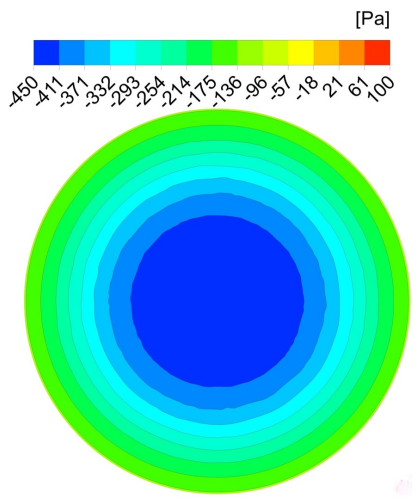

(b) RSM

Figure 14: Contours of static pressure $p$ in pump discharge section computed by steady fine grid $k-\omega \mathrm{SST}$ and RSM analyses (compressed air gauge pressure $p_{m p i}=2 \mathrm{bar}$ ). 


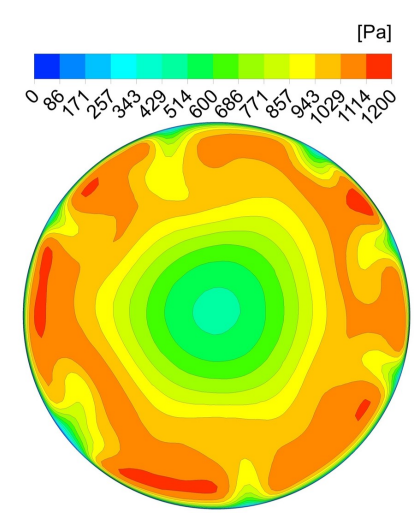

(a) $k-\omega \mathrm{SST}$

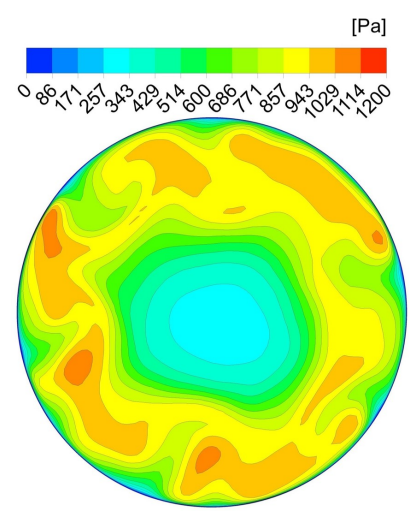

(b) RSM

Figure 15: Contours of total pressure $p^{0}$ in pump discharge section computed by steady fine grid $k-\omega \mathrm{SST}$ and RSM analyses (compressed air gauge pressure $p_{m p i}=2 \mathrm{bar}$ ).

\subsection{AJP fluid dynamics and design}

The results of Subsection 6.1 highlight that the maximum efficiency $\eta$ of the considered multi-nozzle AJP has a relatively low value of about $6.0 \%$ for operation at $p_{m p i}=2$ bar. This section aims at providing design guidelines by investigating the causes for this low efficiency level, and thus envisaging ways to increase it. The analyses focus on the total pressure loss in the jet injection subsystems, and the impact of jet swirl on the overall pump efficiency $\eta$. All discussed investigations are based on steady fine grid SST simulations with $p_{m p i}=2$ bar.

The data of Tab. 4 show that the secondary mass flow rate is about 10 times that of the motive mass flow rate for all considered $p_{m p i}$ values. It is not unusual that the mass flow rate of the entrained fluid be greater than that of the motive fluid, but in jet pumps this often occurs at the expense of severe head losses in the injection subsystem [26]. The motive air of this study's AJP experiences a strong expansion as it enters the bore, hence the higher entrained mass flow rate. It is therefore important to assess the impact of the head losses of the compressed air between the inlet of the compressed air pipe highlighted in the schematic of Fig. 2 and the points of injection in the pump bore. Losses 


\begin{tabular}{||l||l|l|l||l|l|l|l|l||}
\hline \hline Des. & $\begin{array}{l}p_{B}^{0} \\
{[\mathrm{KPa}]}\end{array}$ & $\begin{array}{l}\phi \\
{[\mathrm{mm}]}\end{array}$ & $\begin{array}{l}\beta \\
{\left[{ }^{\circ}\right]}\end{array}$ & $\begin{array}{l}\dot{m}_{\text {s }} \\
{[\mathrm{kg} / \mathrm{s}]}\end{array}$ & $\begin{array}{l}P_{\text {out }} \\
{[\mathrm{W}]}\end{array}$ & $\begin{array}{l}\dot{m}_{p} \\
{[\mathrm{~kg} / \mathrm{s}]}\end{array}$ & $\begin{array}{l}P_{\text {in }} \\
{[\mathrm{W}]}\end{array}$ & $\begin{array}{l}\eta \\
{[\%]}\end{array}$ \\
\hline \hline 1 & 131 & 2.0 & 20 & 0.0754 & 53.6 & 0.01044 & 900.1 & 5.95 \\
\hline 2 & 166 & 3.0 & 20 & 0.1319 & 354.5 & 0.03551 & 2935.1 & 12.08 \\
\hline 3 & 142 & 2.0 & 0 & 0.0841 & 87.4 & 0.01114 & 920.8 & 9.49 \\
\hline
\end{tabular}

Table 5: Mass flows, pressure loss and performance metrics computed with steady fine grid $k-\omega \mathrm{SST}$ analysis for alternative pump designs (compressed air gauge pressure $p_{m p i}=2$ bar).

in this subsystem reduce the effective power available to pump the secondary fluid, and lower the overall efficiency of the device. The losses incurred in the nozzles are investigated by considering the area-averaged total pressure $p_{B}^{0}$ at the discharge hole of each nozzle into the bore. It is found that, for the baseline device, $p_{B}^{0}=131 \mathrm{KPa}$, which corresponds to a loss of gauge total pressure of more than $34 \%$. This $p_{B}^{0}$ value is reported in the row of Tab. 5 labelled 'Des. 1', which stands for Design 1. The next two columns of the table report the nozzle diameter $\phi$ and circumferential inclination angle $\beta$. The five columns in the right portion of the table provide the mass flows of the primary and secondary flows, and the values of $P_{\text {out }}, P_{\text {in }}$ and $\eta$. For Design 1 , these values are provided as quick reference for the analyses of two alternative AJPs below.

To assess the impact of the nozzle diameter on the compressed air total pressure loss and the overall performance of the device, the AJP flow simulation has been carried out increasing the nozzle diameter from $2 \mathrm{~mm}$ (baseline device) to $3.0 \mathrm{~mm}$, which is the configuration named 'Des. 2' in Tab. 5 . Design 2 has $p_{B}^{0}=166 \mathrm{KPa}$, corresponding to a gauge total pressure loss of $17 \%$, half the compressed air head loss of the baseline AJP. The induced alterations of the Design 2 flow field result in its efficiency rising to about 12\%, twice the value of Design 1. This efficiency gain is accompanied by a significant performance gain, as the secondary fluid mass flow increases by $75 \%$ over the value of the baseline AJP. These improvements are triggered by the reduction of the total pressure loss in the nozzles and the larger mass flow rate of compressed air, due also to 
the larger cross section of the nozzles. Also $\dot{m}_{s}$ increases, but the percentage increment of this variable is smaller then that of $\dot{m}_{p}$. Thus, the larger value of $P_{\text {out }}$ with respect to the baseline device is due to a significantly larger energy per unit mass transferred to the secondary flow by the stronger jets of Design 2, which results in higher total pressure at the discharge of Design 2. These findings highlight the importance of optimising the nozzle design, which includes careful choice of the duct diameter, and, when compatible with manufacturing and cost constraints, also the use of variable-section nozzles.

AJP Design 3 has the same nozzles of Design 1, but the jet swirl is removed by setting to zero the nozzle angle $\beta$. Design 3 is found to have $p_{B}^{0}=142 \mathrm{KPa}$, corresponding to a gauge total pressure loss of nearly $30 \%$, slightly smaller than for the baseline AJP, but still significant. Despite this, the features of the Design 3 flow field result in its efficiency rising to about $9.5 \%$. Similarly to the case of Design 2, the efficiency gain of Design 3 is also accompanied by a performance gain, as the secondary fluid mass flow increases by about $11 \%$ over the value of the baseline AJP. Most of the efficiency gain, however, is due to a higher total pressure at the discharge of Design $3 \mathrm{AJP}$, as the ratio $\dot{m}_{s} / \dot{m}_{p}$ of Design 3 is only a few percentage points higher than that of Design 1 AJP. Flow visualisations of the Design 3 flow field, not reported for brevity, show that the jets form a single coaxial jet centred on the duct centreline. This high-speed stream is not wall-bounded and, thus, is not subject to high frictional losses, unlike the high-speed swirling stream of Design 1 . The overall performance improvement achieved by removing the flow swirl, makes one wonder if jet swirl is beneficial to the device performance and efficiency for the pure pumping functionality of the multi-nozzle AJP. Several studies of continuous-jet pumps have compared the performance of devices with no swirl and various levels of swirl intensity produced through swirl generators [10, 11], and efficiency improvements of up to $5 \%$ have been reported for $\beta \approx 15^{\circ}$ [11. Therefore, it seems likely that the level of swirl improving the performance of multi-nozzle AJPs is smaller than that of Design 1 in this study, since the losses due to wall friction shear and higher viscous dissipation of swirling flows with excessively high values of the 
swirl number may outweigh the benefit of promoting entrainment and energising the secondary fluid.

It is expected that even larger performance improvements can be obtained by varying concurrently $\phi$ and $\beta$, and also other design parameters, such as the number of nozzles. All this highlights the possibility of significant improvements the multi-nozzle AJP performance by means of design optimisation.

\section{Conclusions}

RANS CFD simulations using the $k-\omega$ SST and RSM turbulence models have been used to asses the performance and investigate the fluid dynamics of a swirl-inducing multi-nozzle annular jet pump experimentally tested in this research. Validation of the two numerical set-ups was based on pressure tap measurements of the static pressure along the main duct inner wall, and Pitot tube measurements of the static pressure along the main duct centreline for three values of the gauge total pressure of the compressed air generating the swirling jets. The swirl number of the three operating conditions was estimated to be between 0.45 and 0.55 , the range in which the streamline curvature achieves magnitudes requiring the improved modelling capabilities of the RSM analysis. In line with this, the RSM results agreed better than the SST results with the experimental data at the highest gauge total pressure, the regime yielding the largest flow swirl. The RSM and SST analyses were found to get closer as the flow swirl decreased, as expected. Both analyses predicted the same trend of increasing pump performance and efficiency with the compressed air total pressure over the considered range of this variable, although with different values of the performance and efficiency metrics.

Detailed flow analyses highlighted that the flow field downstream of the jet injection features two regions: an annular high-axial speed swirling flow region, and a lower-axial speed central flow region. The SST set-up always predicted lower values of the secondary stream mass flow rate, higher values of the energy per unit mass transferred to the secondary flow, and higher pump 
efficiency. These differences were due to the RSM analysis predicting slightly faster dissipation of the swirling stream energy, lower levels of stream mixing and less penetration of the swirling stream into the pump bore.

The energy efficiency of the baseline pump was about $6 \%$, a relatively low value with respect to the efficiency of other AJPs. Sensitivity analyses aiming at investigating this issue, showed that the pump performance and efficiency are highly sensitive to the nozzle diameter, in that, increasing this parameter from 2 to $3.5 \mathrm{~mm}$ doubled the efficiency due to lower head losses in the nozzles. The flow swirl, controlled by the nozzle axis inclination on the axial direction was also found to notably affect performance and efficiency, since the removal of the flow swirl in the baseline pump increased the efficiency to about $9.5 \%$, highlighting the importance of optimising the nozzle design.

\section{Acknowledgements}

This work was supported by the Lancaster University Centre for Global Eco-Innovation, the European Regional Development Fund, and TCL Cumbria to which the design and manufacture of the multi-nozzle AJP is credited, and

whose CEO Mr. Ian Stephenson is gratefully acknowledged. All project simulations were run on the Lancaster High End Computing (HEC) cluster.

\section{References}

[1] I. Karassik, Pump Handbook, Mechanical engineering, McGraw-Hill, 1976.

[2] O. Kwon, M. Kim, H. Kwon, D. Bae, Two-dimensional numerical simulations on the performance of an annular jet pump, Journal of Visualization 5 (2002) 21-28.

[3] J. E. Bardina, P. G. Huang, T. J. Coakley, Turbulence modeling validation, testing, and development (1997). 
[4] V. Yakhot, S. A. Orszag, S. Thangam, T. B. Gatski, C. G. Speziale, Development of turbulence models for shear flows by a double expansion technique, Physics of Fluids A: Fluid Dynamics 4 (7) (1992) 1510-1520.

[5] N. Namiki, O. Kitamura, M. Yamamoto, Numerical prediction of performance of annular-type jet pump, Nippon Kikai Gakkai Ronbunshu, B Hen/Transactions of the Japan Society of Mechanical Engineers, Part B 63 (607) (1997) 950-956.

[6] M. M. Gibson, B. E. Launder, Ground effects on pressure fluctuations in the atmospheric boundary layer, Journal of Fluid Mechanics 86 (3) (1978) 491-511.

[7] T. Maeda, O. Kitamura, M. Yamamoto, Computation of performance of annular jet pump with and without inlet swirl, in: American Society of Mechanical Engineers, Fluids Engineering Division (Publication) FED, ASME, 1998.

[8] J. Fan, J. Eves, H. Thompson, V. Toropov, N. Kapur, D. Copley, A. Mincher, Computational fluid dynamic analysis and design optimization of jet pumps, Computers and Fluids 46 (1) (2011) 212-217.

[9] M. Xu, X. Yang, X. Long, Q. Lyu, B. Ji, Numerical investigation of turbulent flow coherent structures in annular jet pumps using the LES method, Science China Technological Sciences 61 (1) (2018) 86-97.

[10] Y. Shimizu, S. Nakamura, S. Kuzuhara, S. Kurata, Studies of the configuration and performance of annular type jet pumps, Journal of Fluids Engineering, Transactions of the ASME 109 (3) (1987) 205-212.

[11] D. W. Guillaume, T. A. Judge, Improving the efficiency of a jet pump using a swirling primary jet, Review of Scientific Instruments 75 (2) (2004) $553-555$.

[12] F. Menter, Two-equation Turbulence-models for Engineering Applications, AIAA Journal 32 (8) (1994) 1598-1605. 
[13] Ansys-Inc, Fluent theory guide, Tech. Rep. release 17.2 (2013).

URL https://www . ansys . com/Products/Fluids/ANSYS-Fluent

[14] B. Zhou, B. Fleck, F. Bouak, J. Gauthier, Comparison of swirling effects on ejector performance using four turbulence models, Canadian Aeronautics and Space Journal 46 (4) (2000) 178-182.

[15] S. Hogg, M. Leschziner, Cimputation of highly swirling confined flow with a Reynolds stress turbulence model, Aiaa Journal 27 (1) (1989) 57-63.

[16] M. Leschziner, Modelling engineering flows with reynolds stress turbulence closure, Journal of Wind Engineering and Industrial Aerodynamics 35 (C) (1990) 21-47.

[17] S. Jakirlic, K. Hanjalic, C. Tropea, Modeling Rotating and Swirling Turbulent Flows: A Perpetual Challenge, Aiaa Journal 40 (10) (2002) 1984-1996.

[18] B. E. Launder, G. J. Reece, W. Rodi, Progress in the development of a reynolds-stress turbulence closure, Journal of Fluid Mechanics 68 (3) (1975) $537-566$.

[19] R. Cunningham, Liquid jet pump modelling: Effects of axial dimensions on theory-experiment agreement. (1975) MARCH 24-1975.

[20] H. Sheen, W. Chen, S. Jeng, T. Huang, Correlation of swirl number for a radial-type swirl generator, Experimental Thermal and Fluid Science 12 (4) (1996) 444 - 451. doi:10.1016/0894-1777(95) 00135-2.

[21] A. Gupta, D. Lilley, N. Syred, Swirl Flows, Energy and engineering science series, Abacus Press, 1984.

[22] X. Yang, P. Tucker, Assessment of turbulence model performance: large streamline curvature and integral length scales, Compiters and Fluids 126 (2016) 91-101. 
[23] H. Guo, Z. Chen, C. Yu, Simulation of the effect of geometric parameters on tangentially injected swirling pipe airflow, Computers and Fluids 38 (2009) 1917-1924. doi:10.1016/j.compfluid.2009.05.001.

[24] J. Schetz, U. S. N. Aeronautics, S. Administration, U. of Maryland at College Park, J. H. University, Unified Analysis of Turbulent Jet Mixing, no. v. 1382 in NASA contractor report, National Aeronautics and Space Administration, 1969.

[25] N. H. G. Muller, Water jet pumps, Journal of the Hyrdaulics Division (1975) 83-111.

[26] T. Benjamin, Eductor pumps: The calculations, World Pumps 2001 (2001) 34-38. doi:10.1016/S0262-1762(01)80062-6. 